\title{
A Visual Analytic System for Exploring Consumer Clusters
}

\author{
Ping-Hsuan Huang,,A, Yi-Jheng Huang2,B, Li Huang3,A, Wen-Chieh Lin4,A \\ A Department of Computer Science, National Chiao Tung University \\ B Department of Information Communication, Yuan Ze University \\ ${ }^{1}$ ORCID: 0000-0001-7082-031X, dreammyth9892@gmail.com \\ 2 ORCID: 0000-0003-3036-1483, yjhuang@saturn.yzu.edu.tw \\ 3 ORCID: 0000-0002-6760-1324, backslide.cs06g@nctu.edu.tw \\ 4 ORCID: 0000-0002-9704-5373, wclin@cs.nctu.edu.tw
}

\begin{abstract}
$\underline{\text { Abstract }}$
Consumer transactions analysis is a fundamental component for companies to build strong customer relationships and make good decisions. Visualization can help with such tasks. Existing visualization methods of transaction data analysis often focus on specific purposes, such as abnormal behavior detection and stock analysis. Most of current systems focus on analyzing timevarying transaction pattern and usually focus on analyze webscrape data. Few of them are used to analyze the shopping behavior of customer clusters in physical stores.In this study, we present a visualization system to facilitate the process of transaction data exploration. Our system focuses on functions of customer clustering and exploration of customer characteristics. A distribution view embedded in our system visually demonstrates consumer clustering generated by a dimensional reduction algorithm. The visual clusters allow analysts to explore the characteristics of customers in different clusters. In addition, the correlation hinting method provided by our system automatically highlights overlapping subsets of consumers.It can guide analysts to explore interesting customer clusters. In sum, our system helps analysts find customers with similar behaviors, observe characteristics of interesting subsets, and determine the correlation among data attributes. We validate our system with the consumer transaction data from our collaborating department store. Use cases are provided to show the usability of the system.
\end{abstract}

Keywords: visualization system, coordinated multiple view, dimension reduction, transaction behavior analysis.

\section{Introduction}

Consumer transaction data have been rapidly growing due to the popularity of using computers in business [12]. Numerous companies have utilized data analysis technology to improve their services and attract more customers [13]. Compared with data mining approaches that often only offer final results, visual analytic systems could better fit analysts' needs, because visual systems allow analysts to explore data flexibly and then use their domain knowledge to develop appropriate sales strategies during the exploration process. Many visual analytic systems for analyzing consumer transaction data have been proposed [23]. These visualization systems are often designed for special purposes, such as individual consumption analysis [20], abnormal behavior detection [27] or customer location analysis [18]. Unlike them, our system is designed to explore the shopping patterns of different customer clusters. Although there are some researches also trying to analyze transaction patterns, most of them focus on analyzing time-varying patterns, not the shopping patterns of different customer clusters. Analysts need a system that can help them define different customer clusters, explore the consumer behavior of different clusters, and then they can develop sales strategies for different clusters. 
In this study, we introduce a visualization system for the exploration of consumer clusters (Figure 1). Specifically, our system provides a Distribution View, which allows analysts to conduct dimensional reduction on consumers high-dimensional data and visualize the results in a 2D scatter plot. This view assists analysts to visually detect consumer clusters. Also, we develop a method of Correlation Hinting, which can automatically highlight interesting clusters and guide analysts for further exploration. In addition, our system also provides basic charts that analysts can choose to add to the interface, such as bar charts and ThemeRiver. Analysts can generate charts by selecting the attributes they are interested in, perceive and interact with data via visual representations, and intuitively obtain findings from large complex datasets. Identifying the relationship among multiple aspects of data thus becomes easy with the assistance of multiple dimensions of interactivity combined with coordinated multiple views.

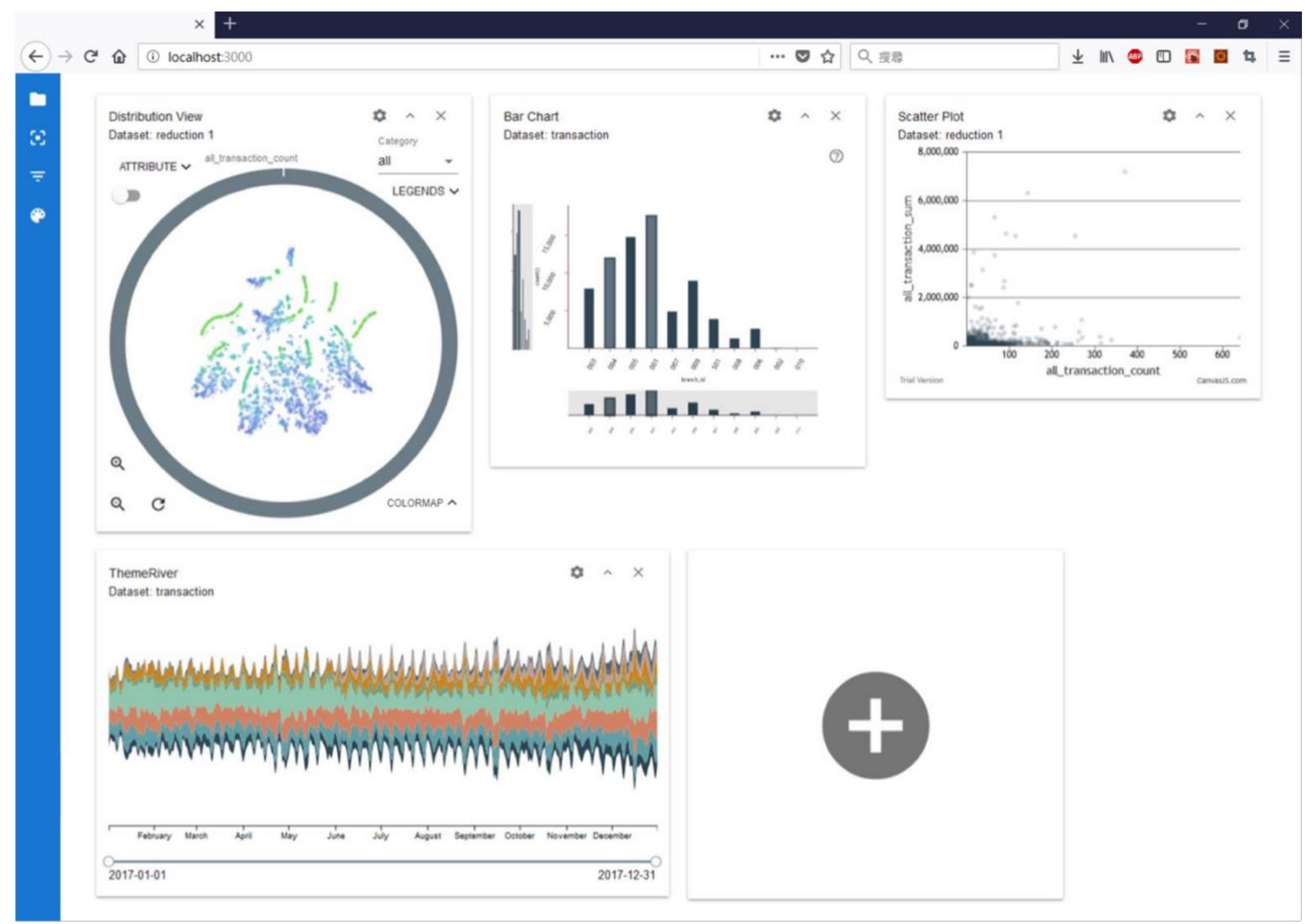

Figure 1. A snapshot of our system.

This study intends to make the following contributions: (1) a visual analytic system that allows analysts to effectively analyze consumer clusters, (2) a distribution view of data scatters that can show the consumer data points under the dimensional reduction and visual abstraction of multivariate data, and (3) a correlation hinting method to guide analysts to explore data.

\section{Related Work}

\subsection{Visual Analysis Approaches for Financial Data}

Useful surveys on visualization system for financial data can be found in [23] and [24]. Many visualization systems are designed to detect anomalies and find frauds as they are the major requirement for existing commerce platforms. Chang et al. [3] presented a set of coordinated 
visualizations based on identifying specific keywords within the wire transactions. Novikova and Kotenko [15] proposed an interactive multi-view approach to describe money transfer activities. The proposed visual representation helps to identify anomalous activities. VAET [17] was a time-series visualization system which can find the representative transaction patterns in the transaction log and identify false consumption behaviors. A visualization system EVA [27] was proposed to support fraud investigation and fine-tune fraud detection algorithms. Didimo et al. [31] drew graph patterns to convey time-varying information for the visual analysis of temporal networks in the fiscal domain, aimed to contrast tax evasion, fiscal frauds, and money laundering. Singh and Best [22] demonstrate that a multi-view visualization system is able to assist auditors in reviewing transaction data. Few years later, they proposed a framework for detection of money laundering activities based on visualization of monetary transactions [32]. Leite et al. [30] proposed NEVA, a visual exploration environment to support the analysis of customer networks in order to reduce false negative and false positive alarms of frauds.

In addition to anomalous activity detection, financial data visualization is also used for other tasks. A common task is the analysis of stock data which contains time series of share prices of companies over a long time, including trend, pattern, performance, and predictive analysis $[11,25]$. Keim et al. [4] used value cells within bar charts to represent business metrics to assist analysts in identifying specific areas. Liu et al. [8] designed and implemented SellTrend based on treemap and timeline to analyze airline travel purchase. Yue et al. [29] presented BitExTract to explore the evolutionary transaction patterns of Bitcoin exchanges from two perspectives: exchange versus exchange and exchange versus client.

Summarizing the above visualization methods, most visualization systems focus on detecting abnormal transactions, while the goal of our system to gain insight into consumer behavior. In addition, previous studies often focus on analyzing time-varying pattern, whereas our system targets consumer clusters and helps analysts explore and analyze the consumption behavior of different consumer clusters.

2.2 Visual Analysis Approaches for Customer Data

Roberts and Laramee [28] provided an excellent review of visualising customer data. Early literature on customer-centric visualisation focused on presenting customer feedback. Broadbeck and Girardin [1] presented a visualisation tool that uses parallel coordinates combined with a tree structure to analyse customer feedback data. Ziegler et al. [5] presented a system that uses clustering techniques to analyse textual customer feedback data from an unspecified online feedback website. Oelke et al. [9] presented a visual analysis of web scraped customer review data using a matrix grid that compares multiple products against a range of features. Wu et al. [10] presented OpinionSeer to visualize customer feedback of hotels. The main focus of this visualisation is the scatter plots in the opinion triangle and the opinion wheel.

With the increasing availability of data from mobile phones, customer-centric visualisation research was shifted to customer behaviour research. Yaeli et al. [18] analysed the movement of customers shopping in retail stores. Shi et al. [16] presented a visual analytics system that tracks user's loyalty of search engine and the behaviour of users switching between different search engines. Dou et al. [19] presented, an analytics system that helps analysts with exploring insight into economic, behavioural and social issues through demographic analysis. Wu et al. [21] used a contour based treemap and a geospatial heat map to present the behavioural patterns in potential customers through the data obtained from China largest telecommunications company.

Our system is also a study to analyze customer behavior. It is worth noting that most of the above-mentioned customer behavior data is retrieved from the Internet, while our customer consumption data comes from physical stores. In addition, above methods usually use algorithms to group customers into some clusters. Their grouping algorithms may refer to previous customer behavior. However, the behavior of customers is always changing, and automatically grouping consumers may limit the possibilities for analysts to explore. On the 
contrary, our system provides a scatter plot to represent every customers using a dimensional reduction algorithm. Analysts can discover and define consumer clusters on their own, without being affected by previous data, and can respond to rapid changes in the market.

\section{Visual analytic system design}

\subsection{Distribution View}

Our system should allow analysts to display high-dimensional data in an easy-to-observe manner to provide an efficient way to demonstrate the characteristics of multivariate data. Therefore, a distribution view is provided (Figure 2). Dimensional reduction algorithm [26] is utilized to reduce the high-dimensional attributes of consumers into 2D space. Analysts can thus discover the relationship of consumer records by observing the visualized clusters generated from dimensional reduction algorithm. In this study, t-distributed stochastic neighborhood embedding ( $\mathrm{t}-\mathrm{SNE}$ ) is utilized as our dimensional reduction algorithm, as this algorithm preserves local distances of high-dimensional data and is particularly suited for visualization. Distribution view consists of two components: scatter and ring.

\subsubsection{Scatter}

We display the reduced-dimensional data in $2 \mathrm{D}$ space, considering that $2 \mathrm{D}$ scatter view remains a highly comprehensible way of displaying distribution on a flat screen [14]. Given the intuitiveness of scattered distribution, we design the interaction of the distribution view as a map; analysts can drag and zoom in and out the map. Analysts can brush points on the view and select these points. The color of the data points can be adjusted as well. Analysts decide the dimension of the color map. Then the system will compute quantiles of the values, map each data point to a group, and color data points with corresponding colors. Figure 2 demonstrates an example of coloring data points. Toggling the right bottom window shows the percentage and value range represented by each color. The quantile groups shown in the window can also be clicked and selected to highlight the consumers in that group.

\subsubsection{Ring}

A ring is added to surround the scattered distribution as a metaphor for a magnifying lens on the map. The ring is designed to demonstrate categorical quantitative information of the data points on the distribution view. We choose ring-like visualization because it is useful in the examination of multivariate data [14].

The ring has no arc and shows $100 \%$ of the data in the view by default. The ring is separated by a number of arcs that display the proportion of the count of data records of specific attributes in raw data with a chosen dimension. An example is shown in Figure 2. Attribute "Gender" is chosen as the observation target, and male consumers, representing the cyan arc in the figure, account for one-fourth in this dataset. We design the outer ring as the quantitative information of selected data for the thickness of the ring in distribution view. The ring will show the proportion of the count of correlated selected data after selecting data records in other views or brushing data points in distribution view, as shown in Figure 2. The length of the arcs and the outer arcs also change by interacting with the scattered distribution, as well as by dragging or zooming in the scattered data records displayed in the range of ring changes. 


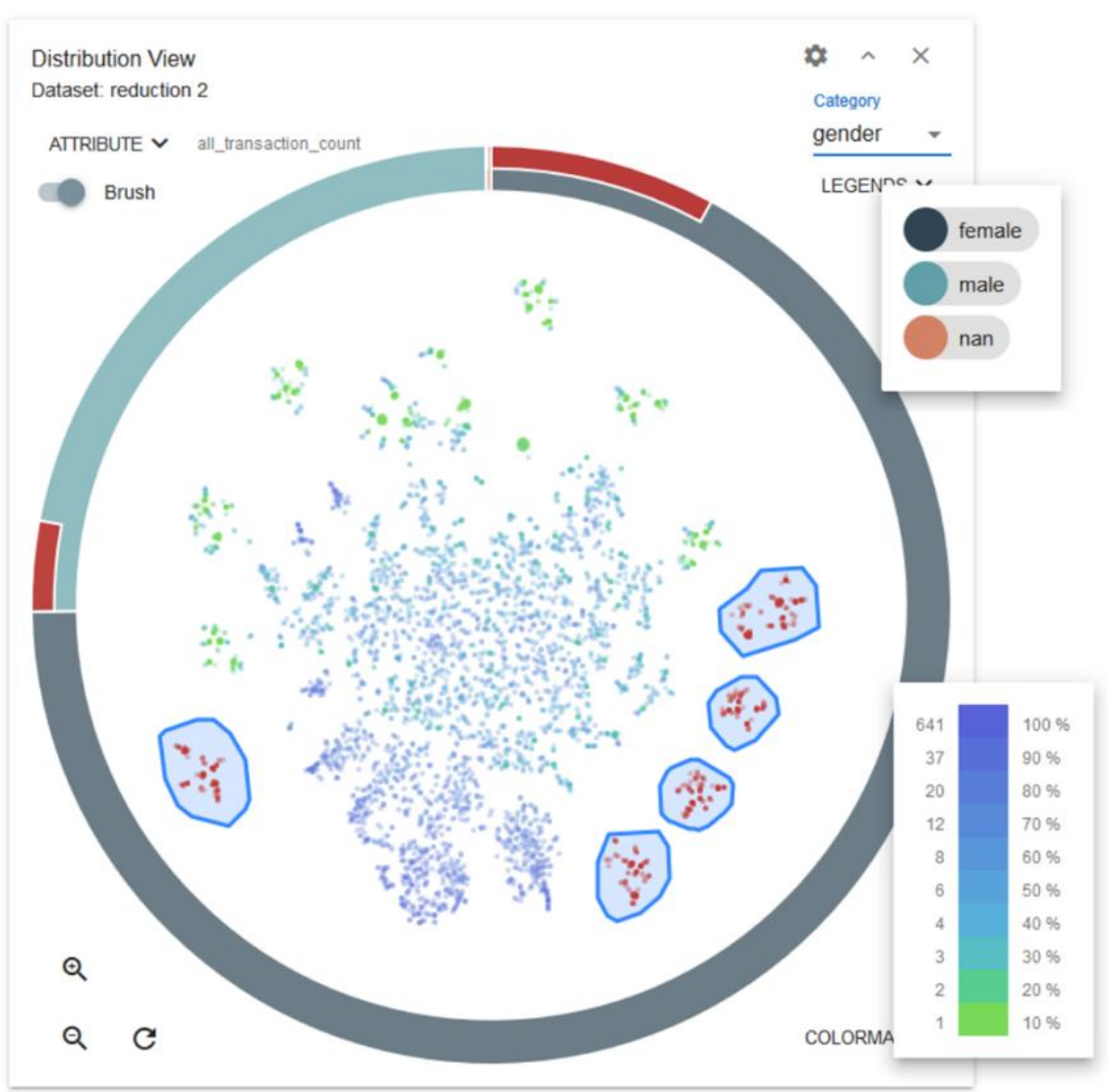

Figure 2. Distribution view.

\subsection{Dynamic Multiple Views}

The design of our system should suit what analysts are familiar with, so we provide some basic charts that analysts can choose to add to the interface, such as bars. To visualize temporal data through time series, our system also provides ThemeRiver. Other types of charts can be easily added to our system. Charts are dynamically and manually added to avoid cluttering caused by multiple views and fit the needs of various analysis. Analysts can choose the data attributes they are interested in and create a relevant chart.

\subsection{Correlation Linking}

Given that dimensional reduction data may not be able to display all the dimensions that analysts are curious about, coordinate multiple view is applied to satisfy the needs of displaying multivariate data and show their correlation by interaction [7]. Brushing and linking [6], as well as focus+context visualization [2], are embedded in the views to support clear visual cognition and intuitive interaction. In addition, our system provides a filtering function to archive current selected data and all its correlated records from other datasets to help analysts focus on the exploration within the part of the data they are interested in.

\subsection{Correlation Hinting}

Our system implements a method of correlation hinting which can automatically provide some hints to help analyst find interesting phenomena. When exploring transaction data, observations of the similarity and dissimilarity of customers is crucial. Finding similar shopping patterns among different customer clusters helps formulate sale strategies. Hence, the concept of correlation hinting means using other charts to map highly correlated consumers. The method is simply designed to find highly overlapping subsets of consumers by grouped subsets in other charts. We implement this function by using bar charts in our system. The method can be easily extended to other charts. Analysts may gain insight into the 
relationship between hinted groups and their correspondent consumers by comparing the hinted groups and the correlated consumers mapped in the distribution view.

We implement two scenarios for the correlation hinting method based on whether analysts have selected several data records. In the case where data are selected, analysts may select a cluster from distribution view and are eager to know "Can this cluster be interpreted by any real-world attribute of interest?" Therefore, we compare the correlated consumer set of selected data and every group in the bar chart and hint the group with the highest ratio. An example computation of correlation linking from a bar chart representing the transaction count of each store is illustrated in Figure 3. If analysts are interested in the hinted bar, then they may select the bar itself to observe the distribution of the selected result and compare the bar with the visual representation of original selected data to find out where the intersected consumers are scattered on the distribution view.

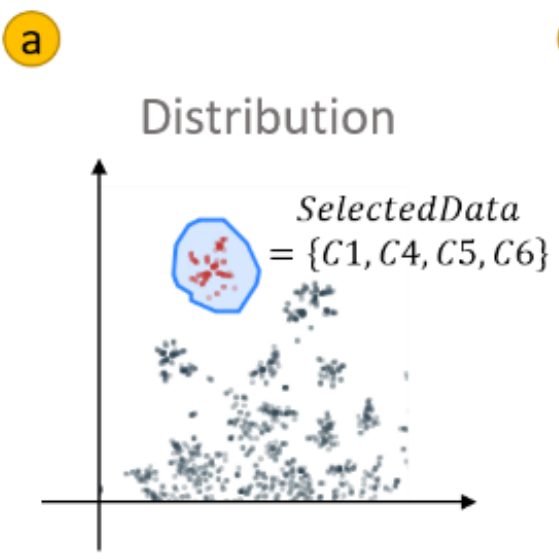

(b) \begin{tabular}{|l|l|l|}
\hline transaction_id & Consumer_id & store_name \\
\hline T00001 & C1 & A \\
\hline T00002 & C2 & A \\
\hline T00003 & C3 & A \\
\hline T00004 & C3 & B \\
\hline T00005 & C4 & B \\
\hline T00006 & C5 & B \\
\hline T00007 & C1 & C \\
\hline T00008 & C2 & C \\
\hline
\end{tabular}

C Transaction count of each Store

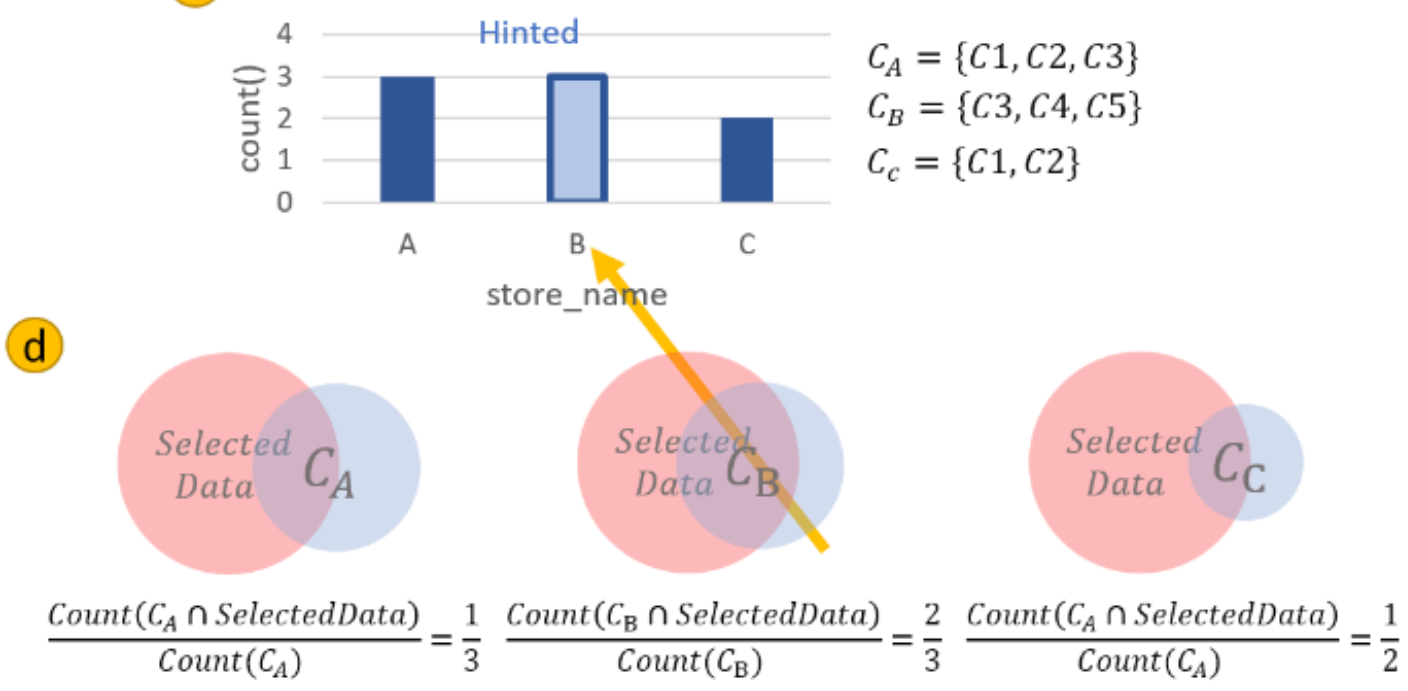

Figure 3. Example of correlation linking method. (a) An analyst selects a cluster he or she is interested in. (b) The raw data of the selected cluster. (c) The cumulative transactions for each store. (d) For each bar, we compute the intersection of selected data and bar set and then hit the bar with the highest overlap ratio. The result of correlation linking implies that the selected consumers trade the most in Store B among the three stores.

In cases without selected data, we compare every two groups in the bar chart and hint the pair with the highest ratio of overlapped correlated consumer sets, which indicates the high likelihood that these groups share the same clusters in distribution view. Analysts may interact with the hinted bars separately to verify their assumption or observe the distribution difference among different groups of correlated consumer sets. The system is able to guide 
analysts to try out some interactions that may lead to interesting findings via this automatic correlation hinting function.

\section{Implementation}

\subsection{Transaction Data}

We use a dataset of customer transactions from our collaborating department store in Taiwan. For privacy and proprietary reasons, private identities have all been encoded. Thus, the phenomenon and data distribution we demonstrate in case studies ate true, whereas the data values are resampled. Our dataset contains the following attributes:

- Consumer information includes the IDs and other information about the consumer who make the transaction, e.g., gender, zip code, and membership level. Note that only the transactions of the members of the department store are recorded.

- Store information contains the IDs and categories of the stores, as well as the branches that the stores belong to. A branch is a large shop containing several different stores, each of which sells different things.

- Transaction information stores the time stamps, consumer and store IDs, and other information about transactions, e.g., the payment amount, whether the consumer preorders, and the number of points the consumer gains through this transaction.

\subsection{System Architecture}

We implement our system as an integrated visualization solution for consumer transaction data analysis. The system utilizes coordinated multiple views with dimensional reduction techniques embedded for observing consumer characteristics, as well as rich interaction, to help data exploration and pattern finding. Three main components exist: data storage and processing, information acquisition, and interface and visualization (Figure 4).

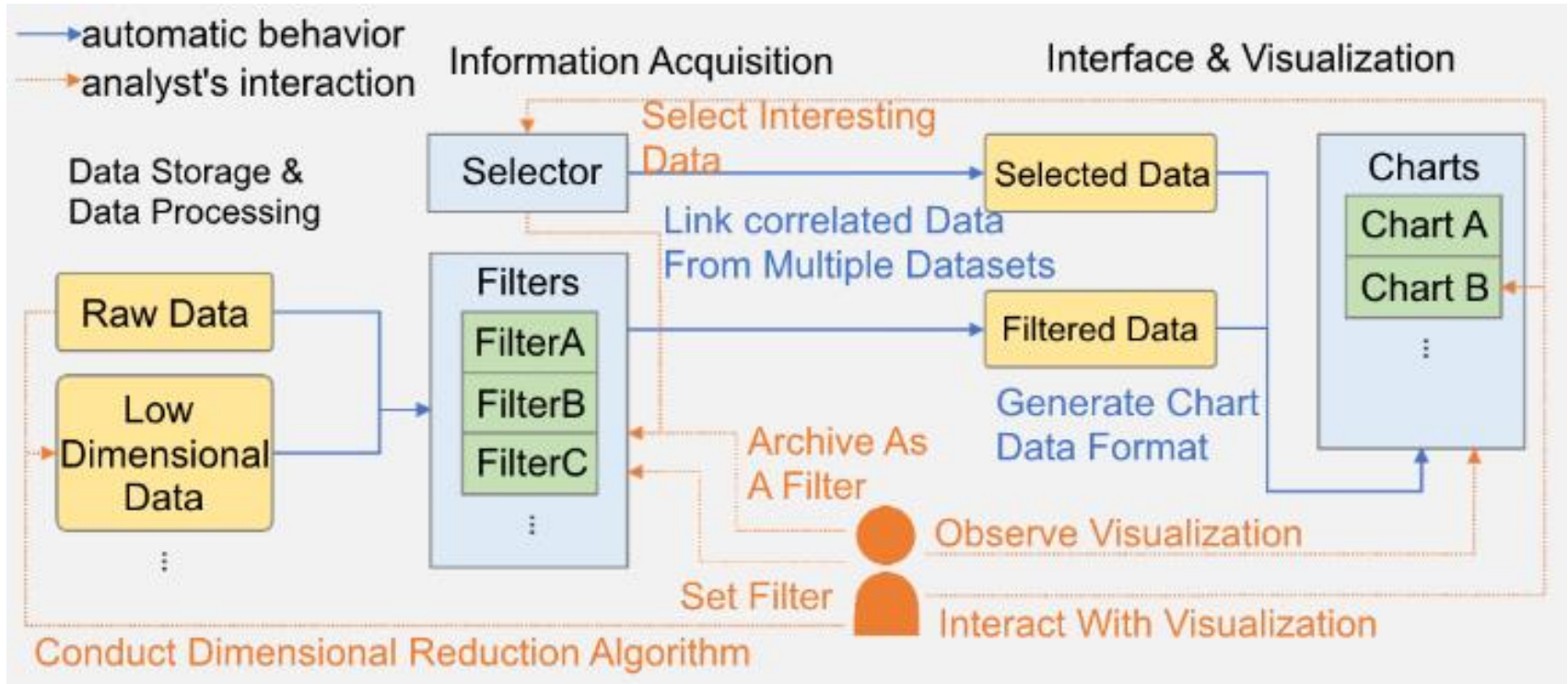

Figure 4. System architecture.

Data Storage and Processing. This component stores raw data and the configuration of dataset correlation. Analysts can also generate new dimensional reduction data by choosing an algorithm and attributes from the user interface.

Information Acquisition. Our coordinated multiple-view system employs selection interaction by brushing and linking techniques to emphasize the correlation within data attributes of multiple datasets. When a visual element is selected, all visual elements related to that record will be synchronized and highlighted at the same time. We define these coordinated data records as selected data. The relationship among visual elements can be direct (e.g., different 
attributes of the same records from the same dataset) or indirect (i.e., correlated data records from different dataset). The relationship of datasets must be configured in advance. The analyst can archive selected data to a filter to further provide the convenience to drill down the data, and all the views will zoom into the subset of filtered data. Analyst may toggle the filters in the list on or off to control what data should be displayed, so that he or she can concentrate on the filtered data.

Interface and Visualization. Interface and visualization is the component responsible for user interface, display, and interactions. Selected and filtered data are passed to each chart to be visualized. The design of the interface and charts can be adjusted based on analyst's preference. Selecting interactions is slightly different within each chart. When an analyst brushes data points from any dataset on view, the brushed data records pass, and the information acquisition component will then generate selected data for this component to update every view.

\subsection{Platform}

Our back-end data processing modules are written using Python 2.7 and scikit-learn. Our server runs via flask. The front-end interface is a single-page web application built by NodeJS. As to front-end framework, we choose ReactJS as our system framework. We use material UI as our library for some of the common components in our user interface, such as grids, tables, and selections. Below are the reasons why ReactJS fits our visualization design and requirement:

- Component-based. Every part in the interface can be divided into components. Given that our interface is designed as a coordinated multiple view system with many different charts, splitting every component apart provides flexibility so that developers can add, modify, or design each chart independently. This flexibility also makes our system extendable because adding any newly designed chart into our system is effortless.

- One-way Data Binding. Data management and real-time updating are also convenient by using ReactJS. Every time an interaction is completed, information acquisition component needs to generate updated selected data to synchronize all coordinated views and pass the data as a state to update every chart. ReactJS will refresh every coordinated visualization by using the updated data.

\section{Case Studies}

The effectiveness of our system is validated in this section. We synthesize a dataset, of which distributions are similar to those of the dataset provided by our collaborating department store, because the data are private and proprietary. The dataset consists of 73,924 transaction records of 4,912 consumer shopping in 10 branches and 1,097 stores. The goal of the analysis is to observe the customers and seek hidden patterns. Please refer to our videos to better understand the operation details of every case.

\subsection{Case 1: Consumers Clustering Based on Branch-wise Transactions}

We start our analysis with the consideration that consumer shopping patterns ought to differ among various branches. We draw a distribution view that performs dimensional reduction on the consumer transaction statistics of all branches, including how many times a customer shopped (transaction count), as well as how much he or she spent in total (total transaction amount) at each branch. As Figure 5 shows, several clusters exist in the distribution view. Then, we add a bar chart showing the transaction count grouped by every branch's ID to infer the common behaviors of the clusters. As shown in Figure 5, we notice that Branches 001 and 004 are hinted, which means that the consumer sets of these two branches have a high ratio of overlapping. A total of 1,425 consumers are revealed to have shopped at both after opening the information window. 


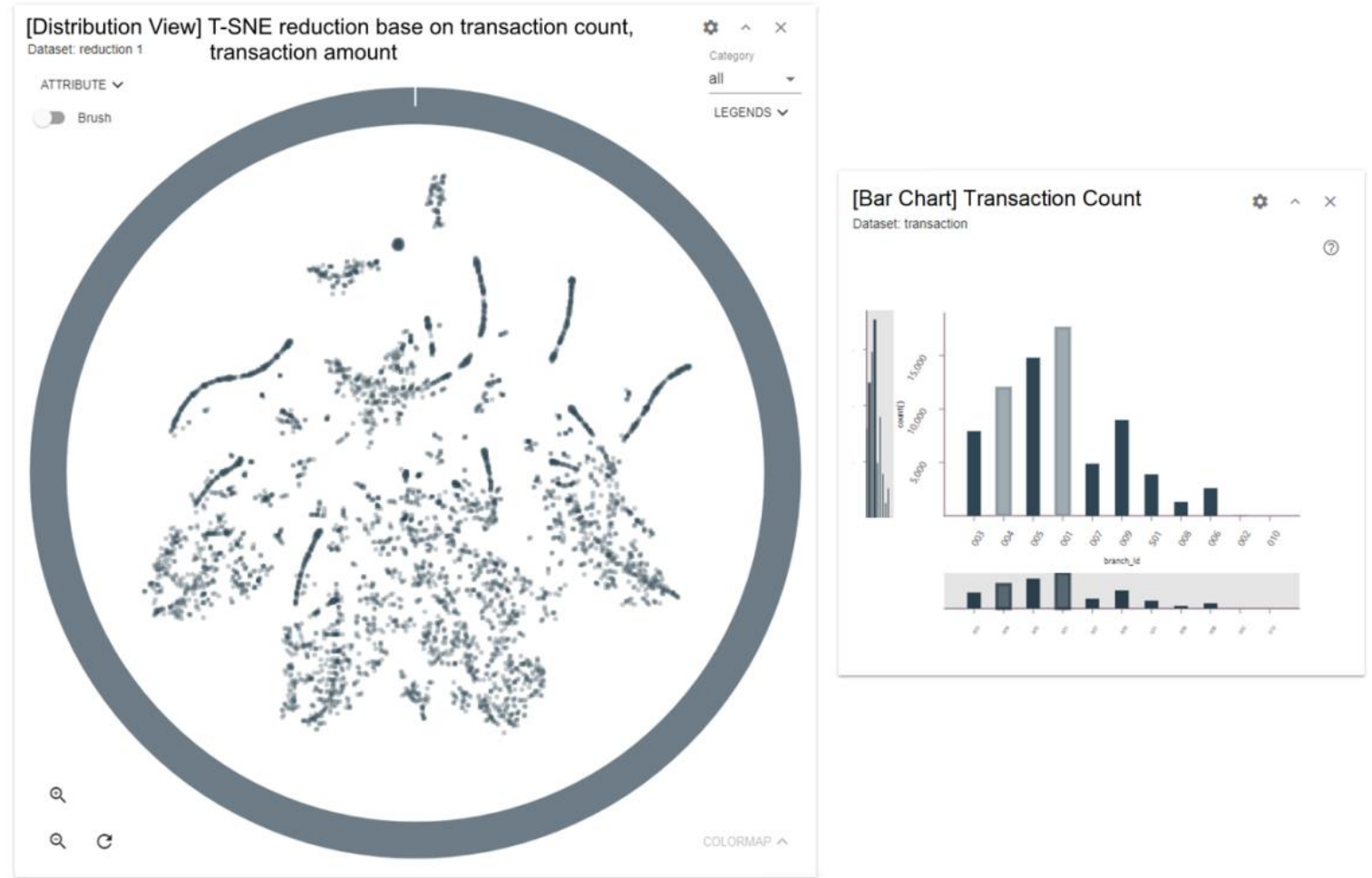

Figure 5. (Left) Distribution view of all consumers; (Right) Transaction count grouped by every branch's ID.

By selecting the two highlighted bars in the bar chart, the bottom right cluster of the data map is highlighted in red (Figures $6[\mathrm{a}]$ and $6[\mathrm{~b}]$ ). We then conclude that the customers in this cluster tend to shop at 001 and 004 . When selecting bars of Branches 001 and 004 , we find that the bar of Branch SO1 is hinted with a thick border in both. Selecting the bar of shopping transactions at Branch So1, we observe the distribution of consumers who have shopped at Branch SO1 is almost inside the cluster 6[d]). The results indicate that the customers of Branch SO1 are a subset of the customers of the cluster.

We also check how the customers at other branches are distributed in the data map by clicking each bar in the chart. Customers of other branches belong to clusters that are highly separated. This finding means that most of the customers shop at a specific branch, except those customers who shop at Branches OO1, OO4, and SO1. When selecting the transactions of Branch 009 (Figure 6[c]), its distribution has partially overlapped with the distributions of several other branches. For instance, the right half parts of the clusters when selecting the transactions of Branches 007 and 009 are similar. A few highlighted data records in the right two clusters also exist, which represents consumers with higher tendency to shop at 001 or O05. This finding suggests that the consumers shopping at Branch 009 are more likely to shop at multiple branches.

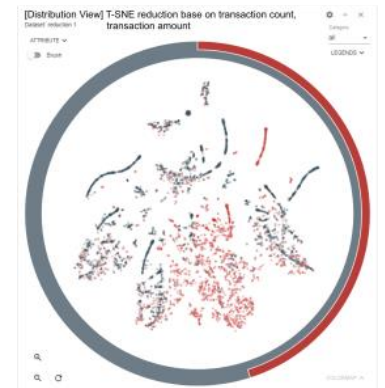

(a) Branch 001

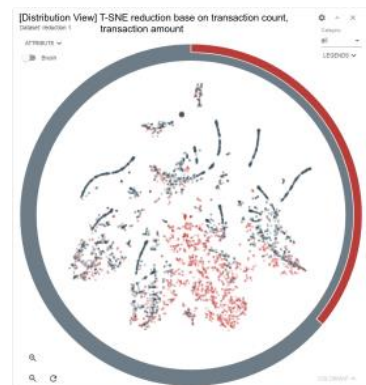

(b) Branch 004

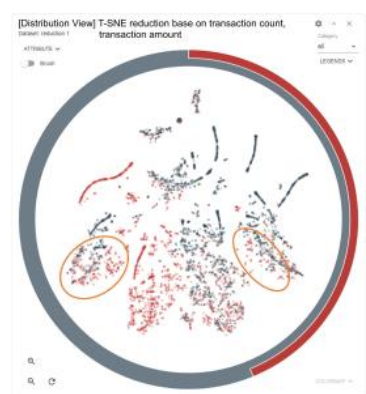

(c) Branch 009

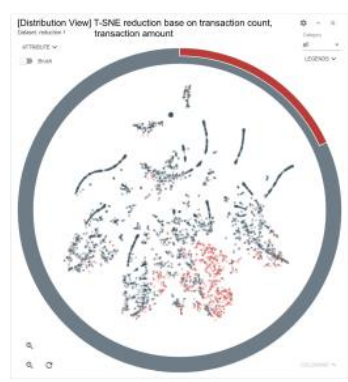

(d) Branch So1

Figure 6. Select transactions of branch (a) 001 , (b) 004 , (c) Oo9, and (d) So1. 


\subsection{Case 2: Consumer Observation of Specific Clusters}

We examine the cluster mentioned in Case 1, which represents customers who have shopped at Branches $0 O 1$ and 004 to demonstrate how analysts can use our system to explore data further. As shown in Figures $7(\mathrm{a})$ and $7(\mathrm{~b})$, we define the biggest cluster of intersected customers of Branches 001 and 004 as Cluster $C_{A}$, and two clusters that are correlated with transactions of Branch 001 but not 004 as $C_{B}$ and $C_{C}$. To analyze the general pattern of these clusters, we change the color map of distribution view such that the consumer data points are colored based on how many times they shopped (transaction count), as well as how much they spent in total (total transaction amount) through the whole year (Figures 7[a] and 7[b]). The distribution view shows that the customers in $C_{A}$ have a large number of total transaction count and total transaction amount over the entire year. These customers shopped more than 12 times in a year with a total transaction amount more than TWD 10,000. By contrast, the consumers in $C_{C}$ have a low transaction count (less than five times in a year), whereas the consumers in $C_{B}$ only shopped once. $C_{B}$ has a large variation in terms of transaction amount. The exact values of transaction count and amount are illustrated by the color map in Figures 7 (a) and $7($ b) , respectively.

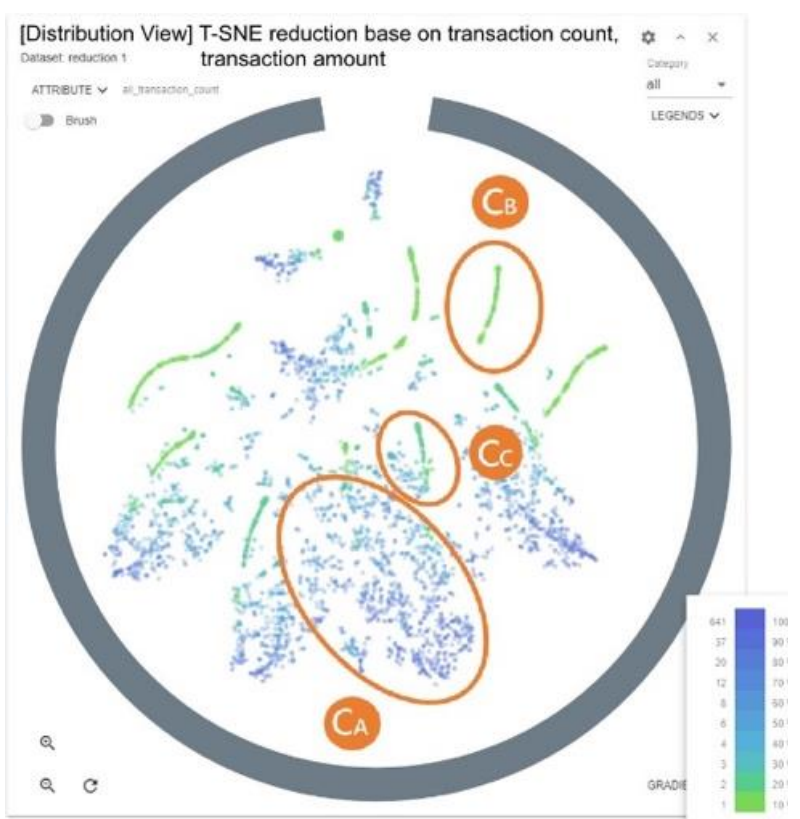

(a) Colored by transaction count

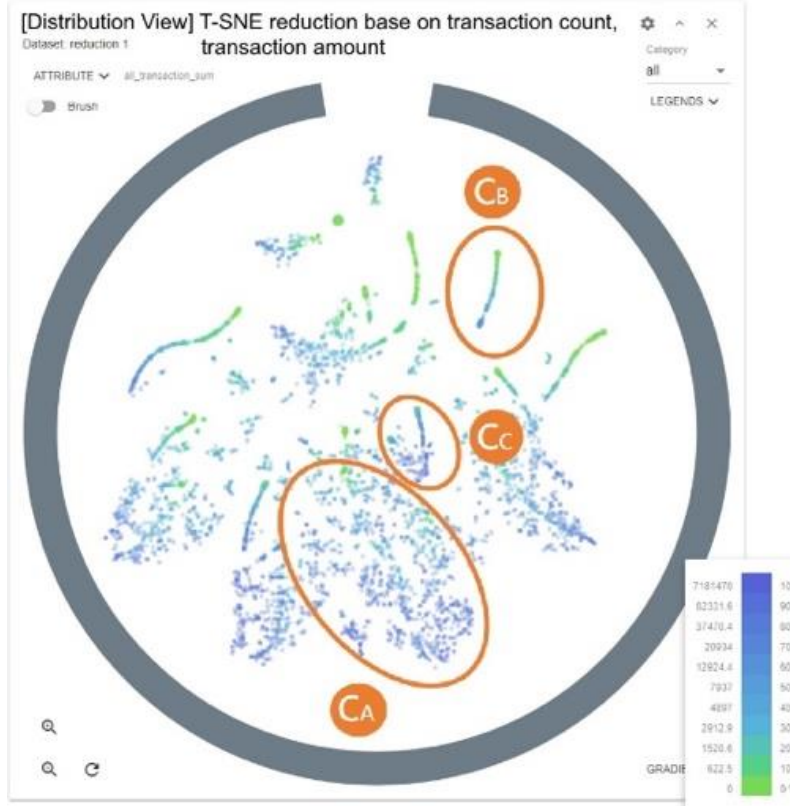

(b) Colored by transaction amount

Figure 7. Color maps and clusters $C_{A}, C_{B}$, and $C_{C}$.

Examine $\mathbf{C}_{\mathbf{B}}$. We closely examine the transactions of these consumers by brushing and filtering $C_{B}$. We then add a bar chart to analyze what kinds of products these consumers have purchased. As shown in Figure 8, those consumers whose total transaction amount in a year is from TWD 8,000 to TWD 20,000 buy at boutiques or luxury brands, whereas those consumers whose transaction amount is between TWD 1,000 and TWD 5,000 buy shoes, fragrance, groceries, and skin care products. Although this cluster represents those consumers who shopped only once or twice at Branch oo1, over $30 \%$ of the consumers purchased luxury goods. 

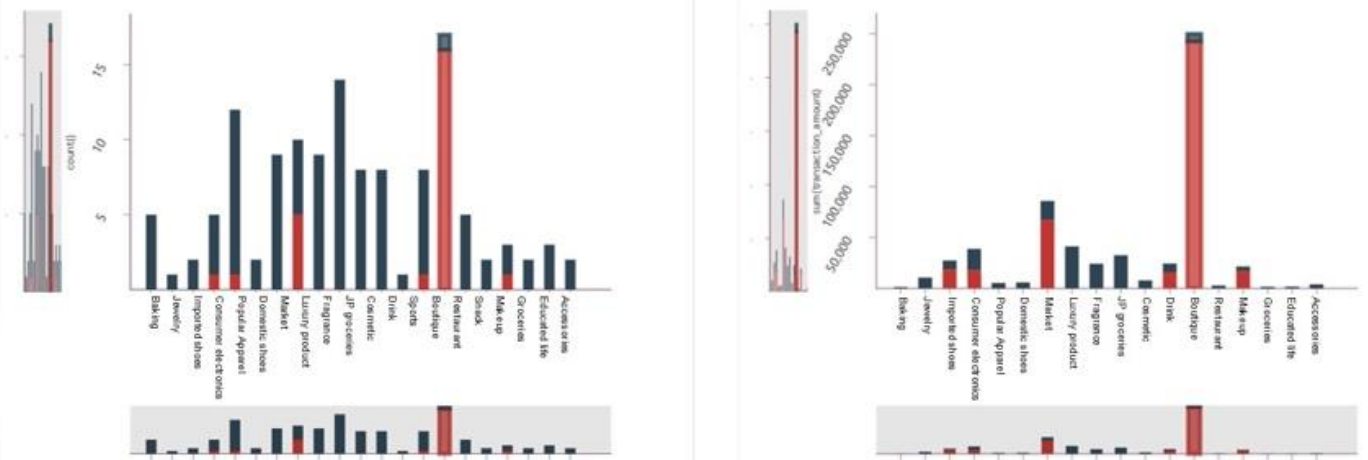

Figure 8. In $C_{B}$, selecting consumers spent TWD 8,000-20,000. They tended to buy boutiques or luxury brands.

Examine $\mathbf{C}_{\mathbf{c}}$. We brush and filter $C_{C}$ and change the color map to view the transaction count and amount of each customer in $C_{C}$. By observing the distribution view, it shows that those consumers who shopped the least (only one transaction in a year) in this cluster surprisingly bought expensive products (more than TWD 50,000). Being curious about the exact amount these consumers spent, we expand the selected panel to check the raw data. The price of the products these consumers bought ranged from TWD 29,800 to TWD 150,000. This finding reveals that these consumers purchased more high-priced products than those in $C_{B}$. Boutiques, luxury brands, jewelry, and watches form the majority of the transaction categories.

Examine $\mathbf{C}_{A}$. The findings in Figure 7 reveal that $C_{A}$ is a cluster with regular customers of Branches 001 and 004 . By filtering $C_{A}$ and adding a bar chart of shopping categories, we find that most of the transactions were done at restaurants and supermarkets, and the categories related to food also accounted for a high percentage of the transactions. In particular, more transactions about edible products exist compared with $C_{B}$ or $C_{C}$. To observe the shopping behavior of the customers in $C_{A}$ who have bought high-priced products, we select and filter the consumers who have shopped in boutiques and for luxury brands, jewelry, and watches, the high-priced products that are also bought with a high tendency in $C_{B}$ and $C_{C}$. Subsequently, we add a ThemeRiver to verify how and when those transactions were done. As shown in Figure 9(a), although these customers had bought these products numerous times, these transactions occur in a specific period of a year, such as anniversary or Christmas sales. To determine consistent shopping behavior, we set the color map to observe the transaction count of these consumers, the ones who have shopped in boutiques and for luxury brands, jewelry, and watches in $C_{A}$. We filter the top $20 \%$ of consumers with high transaction count, who shopped more than 54 times in a year, and find a total of 69 customers. Checking the filtered data, we select each bar in the bar chart and examine their distribution on the ring. We find that almost all of these customers purchased a certain category of products, such as meals, baked goods, and drinks. We then filter these transactions with specific category and add a bar chart of the distribution of stores to check which stores these customers prefer. By choosing the restaurant category, we find that: (1) All 69 customers in this subset have dined in at least one restaurant. (2) Figure 9(b) shows that these customers eat at some specific stores with a pretty high rate within a group of restaurants. 

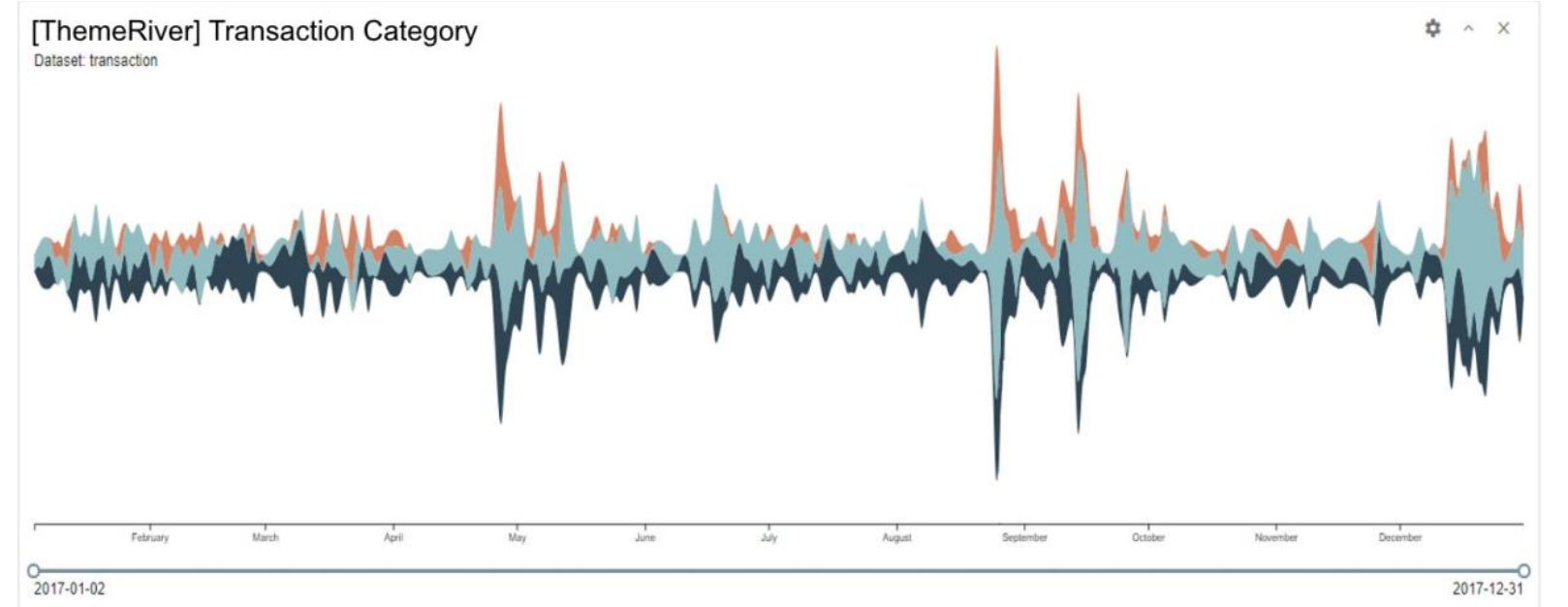

(a)

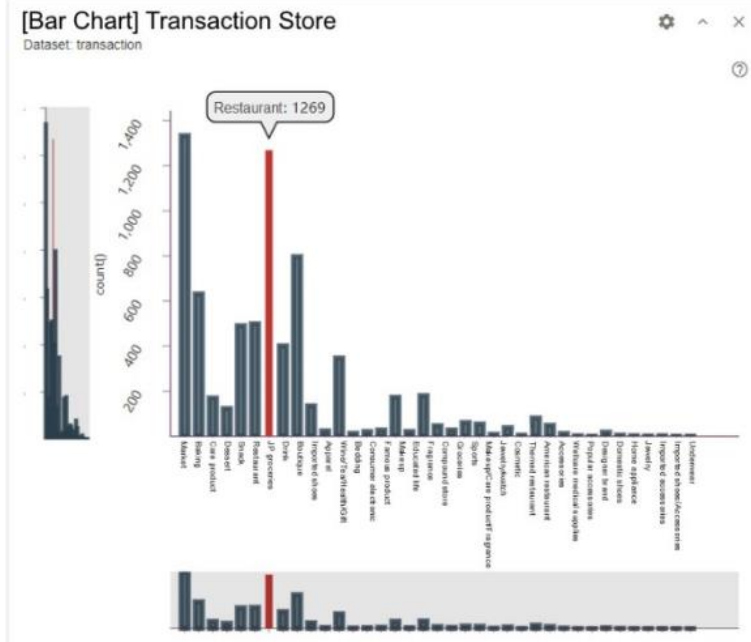

(b)

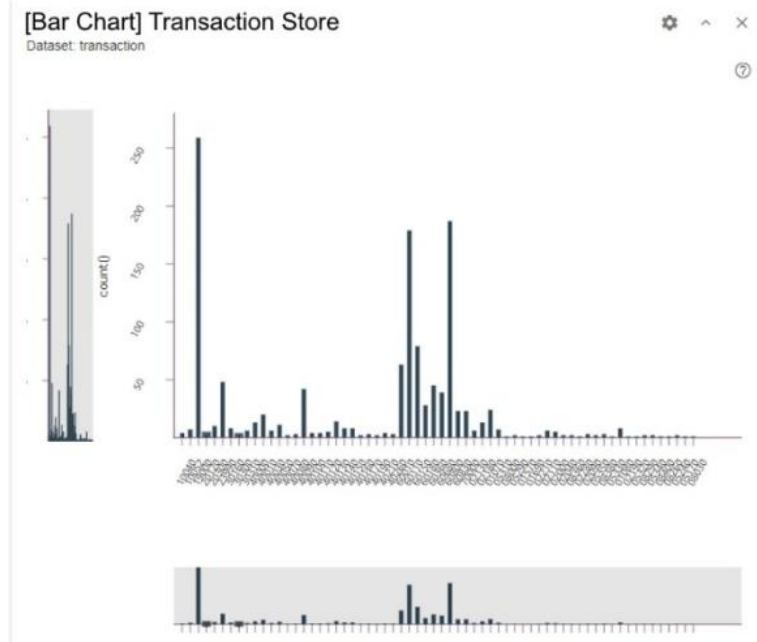

(c)

Figure 9. Observation of consumers in $C_{A}$ who shopped for high-priced products. (a) ThemeRiver of transactions of boutiques, luxury brands, jewelry, and watches. (b) Filtered data as regular consumers in $C_{A}$ who shopped for high-priced products; select transactions of restaurant. (c) Transactions grouped by store ID.

\subsection{Case 3: Analyze Transaction Behavior within Specific Time Range}

We suppose that consumers that shopped at different time ranges have varied preferences or characteristics. To verify our hypothesis, we conduct dimensional reduction algorithm with consumer attributes, such as total amount of transactions, transaction count of each branch, and the shopping percentage of each month. We then demonstrate that our system can assist analysts in understanding consumer behavior during different time periods. We set the colormap as the shopping percentage from January to December to observe the distribution on data map. As presented in Figure 10(a), consumers at the small clusters allocated at the periphery are those with the highest shopping percentage for each month. The clusters of $100 \%$ usually result from consumers shopping once or twice.

Figure 10(a) shows the shopping percentage in May. By dragging the data map to the outer cluster, all colored purple, the ring shows that this cluster has a high percentage of male customers (Figure 10[b]), which is about 1/3. Customers who only shopped in August also have the same sex ratio. Given that almost all the other clusters maintain a stable sex ratio of 1/4, we speculate that this percentage might be due to the celebration of Mother's or Father's Day. 


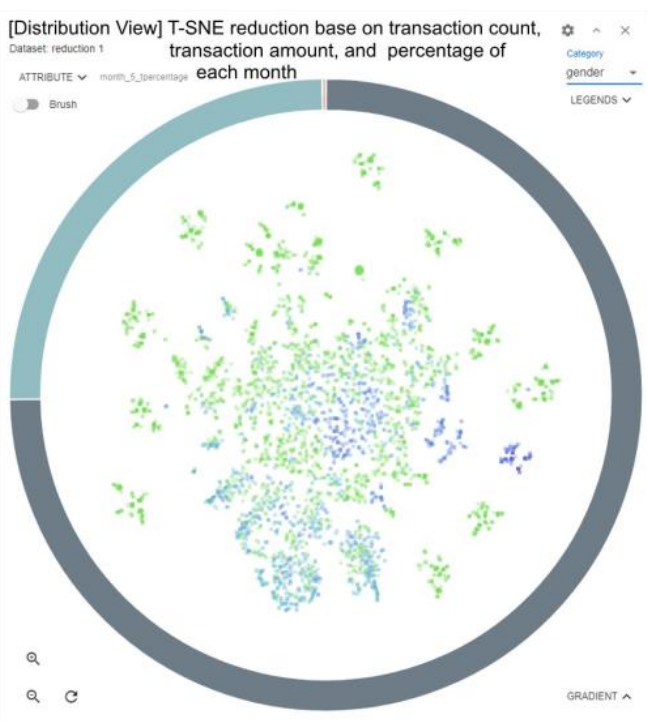

(a) Shopping percentage of May

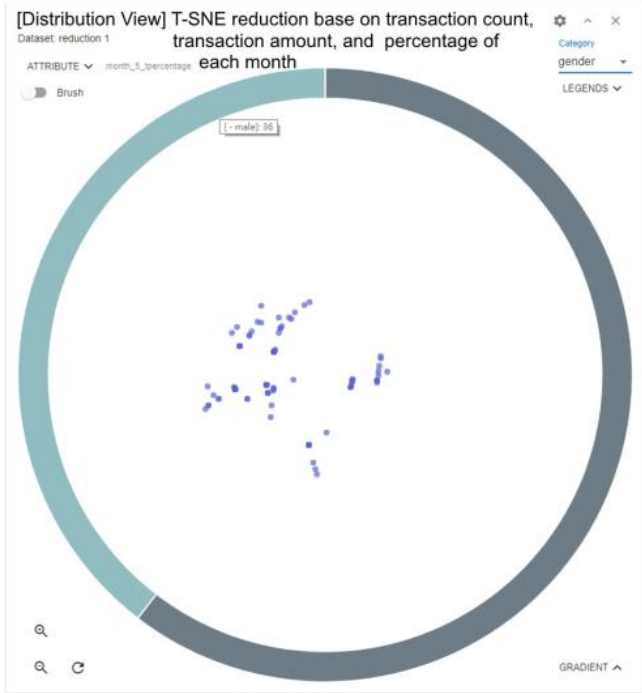

(b) The consumers who only shopped in May

Figure 10. Dimensional view colored by shopping percentage of different months.

We add a ThemeRiver chart to check the transaction count of each branch in a year and determine if any other special temporal behavior exists. As Figure 11(a) shows, the total amount of transactions made in December is slightly higher than that in any other month. Therefore, we observe the ones who have shopped in December and split these customers into three groups based on their shopping percentage. We define the ones who only shopped in December as $F_{A}$, the ones who shopped more than $40 \%$ of his or her transactions in December as $F_{B}$, and the ones who shopped in December but not more than $40 \%$ as $F_{C}$.

The data map and ThemeRiver chart that illustrates transaction count in each branch of these three datasets are displayed in Figures 11(b)(d). The colored data map and ThemeRiver chart clearly show that the shopping behavior differs among these datasets. Focusing on the transactions in December, the consumers of $F_{A}$ shopped more in the last two weekends, whereas the customers of $F_{B}$ liked to shop in the third week (Figures 11[b] and 11[c]). The preference of branches also differs. The category of the stream and its aggregated value of the time period (day or hour) is shown by hovering the cursor over the colored stream of interest. As presented in Figure 11(b), for consumers in $F_{A}$, the yellow stream representing Branch o01 has a boost of transaction count in the third week of December. Another branch with obvious boost is the dark blue stream, representing Branch 009. Compared with the rest of the month, the transaction count increases dramatically in the second and third weekend. Regarding $F_{B}$, the gray stream representing Branch oo6, the transaction count shows a slight improvement in the third week, which is a phenomenon that is not shown in the other two groups. However, the shopping behavior of $F_{C}$ is almost similar to that in other months (Figure 11[d]). Moreover, these consumers shopping records decreased in the third week of December, which is inconsistent with $F_{A}$ and $F_{B}$.

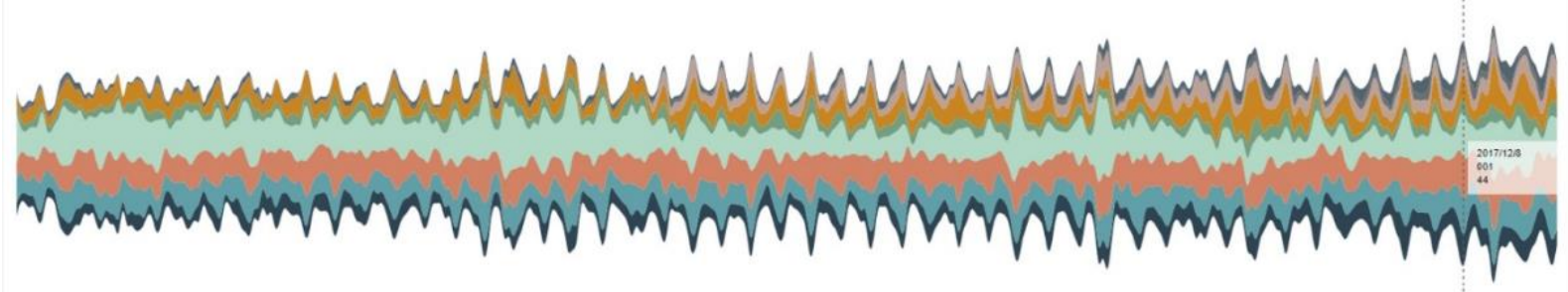

(a) Themeriver of count of transaction of each branch 


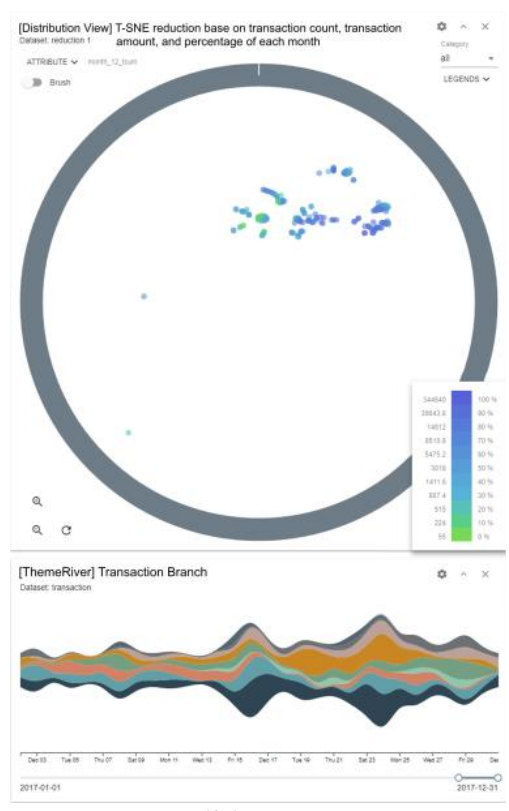

(b) $F_{A}$

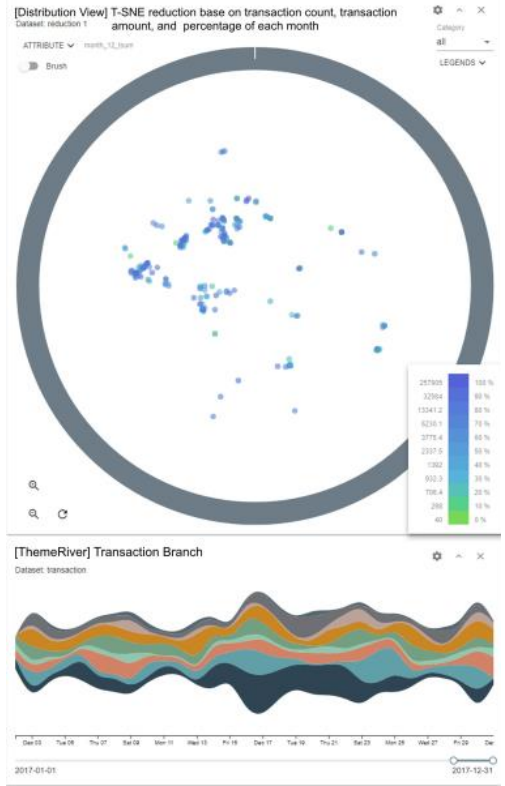

(b) $F_{B}$

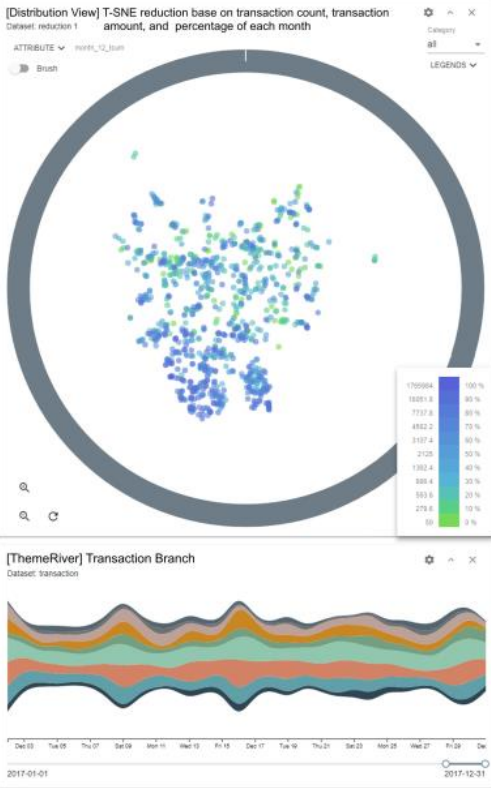

(b) $F_{C}$

Figure 11. Trends from different groups of consumers.

\section{Conclusion, Limitation and Future Work}

In this study, we introduce a visualization system for exploring the shopping behavior of consumer clusters. We design our system with interactive visual analysis features, such as coordinated multiple views and brushing and linking to facilitate the data exploration process of gaining knowledge from consumers' transaction records. Our system also presents the distribution view, a view displaying dimensional reduction data of consumers, to provide analysts with different viewpoints to observe the relationship of consumers and their shopping behaviors. Analysts can observe the correlation among the attributes and drill down the data with the combination of interactions, visualization views provided in distribution view, and correlation linking method. We evaluate the effectiveness and usability of our system using data from a department store chain in Taiwan. The findings from the used cases demonstrate the system's capability of analyzing real-world high-dimensional data.

Among the many topics to be explored in future research, some important ones can be listed as follows. First, Although we implement dimensional reduction algorithm to show the visual abstraction of high dimensional data on distribution view, we did not consider the user scenario and the interactions of other domain-specific data. The specific features of consumer transaction data have not been highlighted in this study. Second, the correlation linking method we proposed has only been used in a bar chart. Given that the concept of hinting the groups with high ratios of intersection is general and intuitive, this concept may be expanded to other kinds of visualization used to display grouped data. More choices might exist to link correlation other than the interaction of subsets. Moreover, the linking methodology should be verified by a rigorous evaluation to prove its effectiveness.

\section{References}

1. Brodbeck, D. \& Girardin, L. Visualization of large-scale customer satisfaction surveys using a parallel coordinate tree in IEEE Symposium on Information Visualization 2003 (IEEE Cat. No. 03TH8714) (2003), 197-201.

2. Novotny, M. \& Hauser, H. Outlierpreserving focus+ context visualization in parallel coordinates. IEEE Transactions on Visualization and Computer Graphics 12, 893-900 (2006). 
3. Chang, R. et al. Wirevis: Visualization of categorical, time-varying data from financial transactions in IEEE Symposium on Visual Analytics Science and Technology (VAST) (2007), 155-162.

4. Keim, D. A., Hao, M. C., Dayal, U. \& Lyons, M. Value-cell bar charts for visualizing large transaction data sets. IEEE transactions on visualization and computer graphics 13, 822-833 (2007).

5. Ziegler, C.-N., Skubacz, M. \& Viermetz, M. Mining and exploring unstructured customer feedback data using language models and treemap visualizations in 2008 IEEE/WIC/ACM International Conference on Web Intelligence and Intelligent Agent Technology 1 (2008), 932-937.

6. Isenberg, P. \& Fisher, D. Collaborative Brushing and Linking for Co-located Visual Analytics of Document Collections in Computer Graphics Forum 28 (2009), 1031-1038.

7. Keefe, D., Ewert, M., Ribarsky, W. \& Chang, R. Interactive coordinated multiple-view visualization of biomechanical motion data. IEEE transactions on visualization and computer graphics 15, 1383-1390 (2009).

8. Liu, Z., Stasko, J. \& Sullivan, T. Selltrend: Inter-attribute visual analysis of temporal transaction data. IEEE Transactions on Visualization and Computer Graphics 15, 10251032 (2009).

9. Oelke, D. et al. Visual opinion analysis of customer feedback data in 2009 IEEE Symposium on Visual Analytics Science and Technology (2009), 187- 194.

10. $\mathrm{Wu}, \mathrm{Y}$. et al. OpinionSeer: interactive visualization of hotel customer feedback. IEEE transactions on visualization and computer graphics 16, 1109- 1118 (2010).

11. Brath, R. \& Macmurchy, P. Spherebased information visualization: Challenges and benefits in 2012 16th International Conference on Information Visualisation (2012), 16.

12. Chen, H., Chiang, R. H. \& Storey, V. C. Business intelligence and analytics: From big data to big impact. MIS quarterly, 1165-1188 (2012).

13. Wang, Y. \& Feng, H. Customer relationship management capabilities. Management Decision (2012).

14. Munzner, T. Visualization Analysis and Design (AK Peters/CRC Press, 2014).

15. Novikova, E. \& Kotenko, I. Visual analytics for detecting anomalous activity in mobile money transfer services in International Conference on Availability, Reliability, and Security (2014), 63-78.

16. Shi, C., Wu, Y., Liu, S., Zhou, H. \& Qu, H. Loyaltracker: Visualizing loyalty dynamics in search engines. IEEE transactions on visualization and computer graphics 20, 17331742 (2014).

17. Xie, C. et al. VAET: A visual analyt ics approach for e-transactions timeseries. IEEE Transactions on Visualization and Computer Graphics 2o, 1743-1752 (2014).

18. Yaeli, A. et al. Understanding customer behavior using indoor location analysis and visualization. IBM Journal of Research and Development 58, 3-1 (2014).

19. Dou, W. et al. DemographicVis: Analyzing demographic information based on user generated content in 2015 IEEE Conference on Visual Analytics Science and Technology (VAST) (2015), 57-64.

20. Hayashi, A., Kohjima, M., Matsubayashi, T. \& Sawada, H. Regularity Measure and Influence Weight for Analysis and Visualization of Consumer's Attitude in International Conference on Information Visualisation (July 2015), 29O-299.

21. $\mathrm{Wu}, \mathrm{W}$. et al. Telcovis: Visual exploration of co-occurrence in urban human mobility based on telco data. IEEE transactions on visualization and computer graphics 22, 935-944 (2015).

22. Gal, G., Singh, K. \& Best, P. Interactive visual analysis of anomalous accounts payable transactions in SAP enterprise systems. Managerial Auditing Journal (2016).

23. Ko, S. et al. A survey on visual analysis approaches for financial data in Computer Graphics Forum 35 (2016), 599- 617. 
24. Rodriguez, J., Kaczmarek, P. \& Depew, D. Visualizing financial data (Wiley Online Library, 2016).

25. Wanner, F. et al. Integrated visual analysis of patterns in time series and text data-workflow and application to financial data analysis. Information Visualization 15, 75-90 (2016).

26. Linderman, G. C., Rachh, M., Hoskins, J. G., Steinerberger, S. \& Kluger, Y. Efficient algorithms for $\mathrm{t}$-distributed stochastic neighborhood embedding. arXiv preprint arXiv:1712.09005 (2017).

27. Leite, R. A. et al. EVA: Visual analytics to identify fraudulent events. IEEE Transactions on Visualization and Computer Graphics 24, 330-339 (2018).

28. Roberts, R. C. \& Laramee, R. S. Visualising business data: A survey. Information 9, 285 (2018).

29. Yue, X. et al. Bitextract: Interactive visualization for extracting bitcoin exchange intelligence. IEEE transactions on visualization and computer graphics 25, 162-171 (2018).

30. A. Leite, R., Gschwandtner, T., Miksch, S., Gstrein, E. \& Kuntner, J. Neva: Visual analytics to identify fraudulent networks in Computer Graphics Forum (2019).

31. Didimo, W., Grilli, L., Liotta, G., Montecchiani, F. \& Pagliuca, D. Visual querying and analysis of temporal fiscal networks. Information Sciences 505, 406-421 (2019).

32. Singh, K. \& Best, P. Anti-Money Laundering: Using data visualization to identify suspicious activity. International Journal of Accounting Information Systems 34, 100418 (2019). 